\title{
Remark on Existence and Uniqueness of Solutions for a Coupled System of Multiterm Nonlinear Fractional Differential Equations
}

\author{
Huichao Zou and Yonghong Fan \\ School of Mathematics and Statistics Science, Ludong University, Yantai, Shandong 264025, China \\ Correspondence should be addressed to Huichao Zou; ldzouhuichao@163.com
}

Received 29 July 2013; Revised 27 November 2013; Accepted 5 December 2013

Academic Editor: Yong Hong Wu

Copyright @ $2013 \mathrm{H}$. Zou and Y. Fan. This is an open access article distributed under the Creative Commons Attribution License, which permits unrestricted use, distribution, and reproduction in any medium, provided the original work is properly cited.

The aim of this paper is to extend the work of Sun et al. (2012) to a more general case for a wider range of function classes of $f$ and g. Our results include the case of the previous work, which are essential improvement of the work of Sun et al. (2012), especially.

\section{Introduction}

Fractional calculus can give a more vivid and accurate description of problems in various fields of sciences than the traditional calculus [1-3]. Recently many complicated dynamic phenomena were modeled by fractional order calculus system and have received more and more attention; see [4-16].

In recent work [12], Sun et al. studied the existence and uniqueness of solutions for a coupled system of multiterm nonlinear fractional differential equations with an initial value condition

$$
\begin{array}{r}
-\mathscr{D}^{\alpha} x(t)=f\left(t, y(t), \mathscr{D}^{\beta_{1}} y(t), \ldots, \mathscr{D}^{\beta_{N}} y(t)\right), \\
\mathscr{D}^{\alpha-i} x(0)=0, \quad i=1,2, \ldots, n_{1}, \\
-\mathscr{D}^{\sigma} y(t)=g\left(t, x(t), \mathscr{D}^{\rho_{1}} x(t), \ldots, \mathscr{D}^{\rho_{N}} x(t)\right), \\
\mathscr{D}^{\sigma-j} y(0)=0, \quad j=1,2, \ldots, n_{2},
\end{array}
$$

where $t \in(0,1], \alpha>\beta_{1}>\beta_{2}>\cdots>\beta_{N}>0, \sigma>\rho_{1}>$ $\rho_{2}>\cdots>\rho_{N}>0, n_{1}=[\alpha]+1, n_{2}=[\sigma]+1$ for $\alpha, \sigma \notin \mathbb{N}$ and $n_{1}=\alpha, n_{2}=\sigma$ for $\alpha, \sigma \in \mathbb{N}, \beta_{q}, \rho_{q}<1$ for any $q \in$ $\{1,2, \ldots, N\}, \mathscr{D}$ is the standard Riemann-Liouville derivative, and $f, g:[0,1] \times \mathbb{R}^{N+1} \rightarrow \mathbb{R}$ are given functions. In order to obtain the existence and uniqueness of solutions of (1), the following growth conditions are introduced in [12].
(H1) There exist two nonnegative functions $a(t), b(t) \in$ $L^{1}[0,1]$ such that

$$
\begin{aligned}
& \left|f\left(t, x_{0}, x_{1}, \ldots, x_{N}\right)\right| \\
& \quad \leq a(t)+c_{0}\left|x_{0}\right|^{\gamma_{0}}+c_{1}\left|x_{1}\right|^{\gamma_{1}}+\cdots+c_{N}\left|x_{N}\right|^{\gamma_{N}}, \\
& \quad\left|G\left(t, x_{0}, x_{1}, \ldots, x_{N}\right)\right| \\
& \quad \leq b(t)+d_{0}\left|x_{0}\right|^{\theta_{0}}+d_{1}\left|x_{1}\right|^{\theta_{1}}+\cdots+d_{N}\left|x_{N}\right|^{\theta_{N}},
\end{aligned}
$$

where $c_{i}, d_{i} \geq 0,0<\gamma_{i}, \theta_{i}<1$ for $i=0,1,2, \ldots, N$.

(H2) The functions $f$ and $g$ satisfy

$$
\begin{gathered}
\left|f\left(t, x_{0}, x_{1}, \ldots, x_{N}\right)\right| \leq c_{0}\left|x_{0}\right|^{\gamma_{0}}+c_{1}\left|x_{1}\right|^{\gamma_{1}}+\cdots+c_{N}\left|x_{N}\right|^{\gamma_{N}}, \\
\left|g\left(t, x_{0}, x_{1}, \ldots, x_{N}\right)\right| \leq d_{0}\left|x_{0}\right|^{\theta_{0}}+d_{1}\left|x_{1}\right|^{\theta_{1}}+\cdots+d_{N}\left|x_{N}\right|^{\theta_{N}},
\end{gathered}
$$

where $c_{i}, d_{i} \geq 0, \gamma_{i}, \theta_{i}>1$ for $i=0,1,2, \ldots, N$.

However, there are many functions which cannot satisfy conditions (H1) and (H2); for example,

$$
g\left(t, x_{0}, x_{1}\right)=\frac{t}{6.08}+\frac{1}{25.26}\left[x_{0}+e^{x_{1}}\right] .
$$

Hence the results of [12] are limited only to a small subset of functions which satisfy (H1) and (H2). This paper thus aims to 
extend the work of Sun et al. [12] to a more general case with more general conditions on $f$ and $g$. Our major contributions of this paper include three aspects.

(1) We extend the function classes to more general case; that is, the power growth assumptions (H1) and $(\mathrm{H} 2)$ are replaced by a very general assumption where the functions $\phi\left(\left|x_{0}\right|,\left|x_{1}\right|, \ldots,\left|x_{N}\right|\right)$ and $\psi\left(\left|x_{0}\right|,\left|x_{1}\right|\right.$, $\left.\ldots,\left|x_{N}\right|\right)$ are only required to be nondecreasing function classes (see (A1)), which implies that the function classes are extended to more general case and also include the case of [12] as a special case. In mathematics and applied science, this generalization is important and interesting.

(2) In [12], the weight functions considered constants $c_{0}, c_{1}, \ldots, c_{N}$. But in physics, the influence of weight functions for the whole system is important, so in this work, we improve the weight functions to general Lebesgue integral functions $b(t), d(t) \in L^{1}[0,1]$, which is also an essential improvement.

(3) In this paper, the nonlinearities $f$ and $g$ are allowed to be exponential growth. However, in [12], the nonlinearities $f$ and $g$ are only allowed to be power growth. It is known that in most cases exponential growth is faster than power growth. From this aspect, this is also a major contribution of this paper.

The remaining part of the paper is organized as follows. In Section 2, some preliminary results including definitions, notations, and lemmas are given. Section 3 presents the main results and the proof of the results. In addition, an example is given to illustrate the application of the main results.

\section{Preliminaries and Lemmas}

Definition 1 (see [1-3]). The fractional integral of order $\alpha>0$ of a function $x:(a,+\infty) \rightarrow R$ is given by

$$
I^{\alpha} x(t)=\frac{1}{\Gamma(\alpha)} \int_{a}^{t}(t-s)^{\alpha-1} x(s) d s,
$$

provided that the right-hand side is pointwisely defined on $(a,+\infty)$.

Definition 2 (see [1-3]). The Riemann-Liouville fractional derivative of order $\alpha>0$ of a function $x:(a,+\infty) \rightarrow R$ is given by

$$
\mathscr{D}^{\alpha} x(t)=\frac{1}{\Gamma(n-\alpha)}\left(\frac{d}{d t}\right)^{n} \int_{a}^{t}(t-s)^{n-\alpha-1} x(s) d s,
$$

where $n=[\alpha]+1,[\alpha]$ denotes the integer part of the number $\alpha$, and $t>a$, provided that the right-hand side is defined on $(a,+\infty)$.

Lemma 3 (see [1]). Assume that $x \in L^{1}[0,1]$ with a fractional derivative of order $\alpha>0$; then

$$
I^{\alpha} \mathscr{D}^{\alpha} x(t)=x(t)+c_{1} t^{\alpha-1}+c_{2} t^{\alpha-2}+\cdots+c_{n} t^{\alpha-n},
$$

where $c_{i} \in R, i=1,2, \ldots, n, n=[\alpha]+1$.
Lemma 4 (see [12]). Suppose that $h \in L^{1}[0,1]$. Then the initial value problem

$$
\begin{gathered}
\mathscr{D}^{\alpha} x(t)=h(t), \quad \alpha>0, t \in[a, b], \\
\mathscr{D}^{\alpha} x(a)=b_{k}, \quad k=1,2, \ldots n,
\end{gathered}
$$

has a unique solution

$$
x(t)=\sum_{j=1}^{n} \frac{b_{j}}{\Gamma(\alpha-j+1)}(t-a)^{\alpha-j}+\frac{1}{\Gamma(\alpha)} \int_{a}^{t} \frac{h(s)}{(t-s)^{1-\alpha}} d s
$$

where $n=[\alpha]+1$ for $\alpha \notin \mathbb{N}$ and $\alpha=n$ for $\alpha \in \mathbb{N}$.

Let $I=[0,1]$ and let $C(I)$ be the space of all continuous functions defined on $I$. We define the space

$$
\begin{aligned}
X \times Y=\{ & (x, y) \mid(x, y) \in C(I) \times C(I), \\
& \left(\mathscr{D}^{\rho_{j}} x(t), \mathscr{D}^{\beta_{j}} y(t)\right) \in C(I) \\
& \times C(I), j=1,2, \ldots, N\}
\end{aligned}
$$

endowed with the norm $\|(x, y)\|_{X \times Y}=\max \left\{\|x\|_{X},\|y\|_{Y}\right\}$, where

$$
\begin{aligned}
& \|x\|_{X}=\max _{t \in I}|x(t)|+\sum_{j=1}^{N} \max _{t \in I}\left|\mathscr{D}^{\rho_{j}} x(t)\right|, \\
& \|y\|_{Y}=\max _{t \in I}|y(t)|+\sum_{j=1}^{N} \max _{t \in I}\left|\mathscr{D}^{\beta_{j}} y(t)\right| .
\end{aligned}
$$

Then $X \times Y$ is a Banach space with norm $\|(x, y)\|_{X \times Y}$.

By Lemma 4, system (1) is equivalent to the following coupled system of integral equations:

$x(t)$

$=\frac{1}{\Gamma(\alpha)} \int_{0}^{t}(t-s)^{\alpha-1} f\left(s, y(s), \mathscr{D}^{\beta_{1}} y(s), \ldots, \mathscr{D}^{\beta_{N}} y(s)\right) d s$,

$y(t)$

$=\frac{1}{\Gamma(\sigma)} \int_{0}^{t}(t-s)^{\sigma-1} g\left(s, x(s), \mathscr{D}^{\rho_{1}} x(s), \ldots, \mathscr{D}^{\rho_{N}} x(s)\right) d s$.

Define an operator $T: X \times Y \rightarrow X \times Y$

$$
\begin{aligned}
& T(x, y)(t) \\
& =\left(T_{1} x(t), T_{2} y(t)\right) \\
& =\left(\frac{1}{\Gamma(\alpha)} \int_{0}^{t}(t-s)^{\alpha-1}\right. \\
& \quad \times f\left(s, y(s), \mathscr{D}^{\beta_{1}} y(s), \ldots, \mathscr{D}^{\beta_{N}} y(s)\right) d s,
\end{aligned}
$$




$$
\begin{aligned}
& \frac{1}{\Gamma(\sigma)} \int_{0}^{t}(t-s)^{\sigma-1} \\
& \left.\quad \times g\left(s, x(s), \mathscr{D}^{\rho_{1}} x(s), \ldots, \mathscr{D}^{\rho_{N}} x(s)\right) d s\right) .
\end{aligned}
$$

It is obvious that a fixed point of operator $T$ is the solution of coupled system (1).

\section{Main Result}

Theorem 5. Let $f, g:[0,1] \times \mathbb{R}^{N+1} \rightarrow \mathbb{R}$ be continuous. Assume that

(A1) there exist nonnegative functions $a, b, c, d \in L^{1}[0,1]$ and nonnegative nondecreasing functions $\phi, \psi$ with respect to each variable $x_{i}, i=0,1,2, \ldots, N$, such that $\left|f\left(t, x_{0}, x_{1}, \ldots, x_{N}\right)\right| \leq a(t)+b(t) \phi\left(\left|x_{0}\right|,\left|x_{1}\right|, \ldots,\left|x_{N}\right|\right)$, $\left|g\left(t, x_{0}, x_{1}, \ldots, x_{N}\right)\right| \leq c(t)+d(t) \psi\left(\left|x_{0}\right|,\left|x_{1}\right|, \ldots,\left|x_{N}\right|\right)$;

(A2) there exists a constant $R_{0}>\max \left\{k_{1}, l_{1}\right\}$ such that

$$
\begin{aligned}
& \phi\left(R_{0}, R_{0}, \ldots, R_{0}\right) \leq \frac{R_{0}-k_{1}}{k_{2}}, \\
& \psi\left(R_{0}, R_{0}, \ldots, R_{0}\right) \leq \frac{R_{0}-l_{1}}{l_{2}}, \\
& k_{1}=\max _{t \in I}\left(\frac{1}{\Gamma(\alpha)} \int_{0}^{t}(t-s)^{\alpha-1}|a(s)| d s\right. \\
& \left.+\sum_{j=1}^{N} \frac{1}{\Gamma\left(\alpha-\rho_{j}\right)} \int_{0}^{t}(t-s)^{\alpha-\rho_{j}-1}|a(s)| d s\right), \\
& k_{2}=\max _{t \in I}\left(\frac{1}{\Gamma(\alpha)} \int_{0}^{t}(t-s)^{\alpha-1}|b(s)| d s\right. \\
& \left.+\sum_{j=1}^{N} \frac{1}{\Gamma\left(\alpha-\rho_{j}\right)} \int_{0}^{t}(t-s)^{\alpha-\rho_{j}-1}|b(s)| d s\right), \\
& l_{1}=\max _{t \in I}\left(\frac{1}{\Gamma(\sigma)} \int_{0}^{t}(t-s)^{\sigma-1}|c(s)| d s\right. \\
& \left.+\sum_{j=1}^{N} \frac{1}{\Gamma\left(\sigma-\beta_{j}\right)} \int_{0}^{t}(t-s)^{\sigma-\beta_{j}-1}|c(s)| d s\right), \\
& l_{2}=\max _{t \in I}\left(\frac{1}{\Gamma(\sigma)} \int_{0}^{t}(t-s)^{\sigma-1}|d(s)| d s\right. \\
& \left.+\sum_{j=1}^{N} \frac{1}{\Gamma\left(\sigma-\beta_{j}\right)} \int_{0}^{t}(t-s)^{\sigma-\beta_{j}-1}|d(s)| d s\right) .
\end{aligned}
$$

Then the coupled system (1) has a solution.
Proof. Define a closed ball of Banach space $X \times Y$

$$
B=\left\{(x, y) \in X \times Y:\|(x, y)\|_{X \times Y} \leq R_{0}\right\} .
$$

We will prove that $T: B \rightarrow B$. In fact, for any $(x, y) \in B$, by (A1), we have

$$
\begin{aligned}
\left|T_{1} x(t)\right| & \left|\frac{1}{\Gamma(\alpha)} \int_{0}^{t}(t-s)^{\alpha-1} f\left(s, y(s), \mathscr{D}^{\beta_{1}} y(s), \ldots, \mathscr{D}^{\beta_{N}} y(s)\right) d s\right| \\
\leq & \frac{1}{\Gamma(\alpha)} \int_{0}^{t}(t-s)^{\alpha-1}|a(s)| d s \\
& +\frac{\phi\left(R_{0}, R_{0}, \ldots, R_{0}\right)}{\Gamma(\alpha)} \int_{0}^{t}(t-s)^{\alpha-1}|b(s)| d s, \\
\left|\mathscr{D}^{\rho_{j}} T_{1} x(t)\right| & \left|\mathscr{D}^{\rho_{j}} I^{\alpha} f\left(t, y(t), \mathscr{D}^{\beta_{1}} y(t), \ldots, \mathscr{D}^{\beta_{N}} y(t)\right)\right| \\
= & \left|I^{\alpha-\rho_{j}} f\left(t, y(t), \mathscr{D}^{\beta_{1}} y(t), \ldots, \mathscr{D}^{\beta_{N}} y(t)\right)\right| \\
= & \frac{1}{\Gamma\left(\alpha-\rho_{j}\right)} \\
& +\frac{\phi\left(R_{0}, R_{0}, \ldots, R_{0}\right)}{\Gamma\left(\alpha-\rho_{j}\right)} \int_{0}^{t}(t-s)^{\alpha-\rho_{j}-1}|b(s)| d s . \\
& \times \int_{0}^{t}(t-s)^{\alpha-\rho_{j}-1} f\left(s, y(s), \mathscr{D}^{\beta_{1}} y(s), \ldots, \mathscr{D}^{\beta_{N}} y(s)\right) d s \\
\leq & \frac{1}{\Gamma\left(\alpha-\rho_{j}\right)} \\
& \times \int_{0}^{t}(t-s)^{\alpha-\rho_{j}-1}|a(s)| d s
\end{aligned}
$$

Thus it follows from (18) and (A2) that

$$
\begin{aligned}
\left\|T_{1} x\right\|_{X} & =\max _{t \in I}\left|T_{1} x(t)\right|+\sum_{j=1}^{N} \max _{t \in I}\left|\mathscr{D}^{\rho_{j}} T_{1} x(t)\right| \\
& \leq k_{1}+k_{2} \phi\left(R_{0}, R_{0}, \ldots, R_{0}\right) \leq R_{0} .
\end{aligned}
$$

In the same way, we also have

$$
\begin{aligned}
\left\|T_{2} y\right\|_{Y} & =\max _{t \in I}\left|T_{2} y(t)\right|+\sum_{j=1}^{N} \max _{t \in I}\left|\mathscr{D}^{\rho_{j}} T_{2} y(t)\right| \\
& \leq l_{1}+l_{2} \psi\left(R_{0}, R_{0}, \ldots, R_{0}\right) \leq R_{0} .
\end{aligned}
$$

Consequently, $\left\|T_{1} x\right\|_{X} \leq R_{0}$ and $\left\|T_{2} y\right\|_{Y} \leq R_{0}$, and then $\|T\|_{X \times Y} \leq R_{0}$ for any $(x, y) \in B$; that is, $T: B \rightarrow B$.

By [12], we know that the operator $T$ is completely continuous. Therefore, the Schauder fixed point theorem implies that coupled system (1) has a solution in $B$. The proof is completed. 
From Theorem 5, we easily obtain the following corollaries.

Corollary 6. Let $f, g:[0,1] \times \mathbb{R}^{N+1} \rightarrow \mathbb{R}$ be continuous. Assume that

(A1) there exist nonnegative functions $c, d \in L^{1}[0,1]$ and nonnegative nondecreasing functions $\phi, \psi$ with respect to each variable $x_{i}, i=0,1,2, \ldots, N$, such that

$$
\begin{aligned}
& \left|f\left(t, x_{0}, x_{1}, \ldots, x_{N}\right)\right| \leq b(t) \phi\left(\left|x_{0}\right|,\left|x_{1}\right|, \ldots,\left|x_{N}\right|\right), \\
& \left|g\left(t, x_{0}, x_{1}, \ldots, x_{N}\right)\right| \leq d(t) \psi\left(\left|x_{0}\right|,\left|x_{1}\right|, \ldots,\left|x_{N}\right|\right) ;
\end{aligned}
$$

(A2) there exists a positive constant $R_{0}$ such that

$$
\begin{gathered}
\phi\left(R_{0}, R_{0}, \ldots, R_{0}\right) \leq \frac{R_{0}}{k_{2}}, \\
\psi\left(R_{0}, R_{0}, \ldots, R_{0}\right) \leq \frac{R_{0}}{l_{2}},
\end{gathered}
$$

where

$$
\begin{aligned}
& k_{2}=\max _{t \in I}( \frac{1}{\Gamma(\alpha)} \int_{0}^{t}(t-s)^{\alpha-1}|b(s)| d s \\
&\left.+\sum_{j=1}^{N} \frac{1}{\Gamma\left(\alpha-\rho_{j}\right)} \int_{0}^{t}(t-s)^{\alpha-\rho_{j}-1}|b(s)| d s\right), \\
& l_{2}=\max _{t \in I}\left(\frac{1}{\Gamma(\sigma)} \int_{0}^{t}(t-s)^{\sigma-1}|d(s)| d s\right. \\
&\left.+\sum_{j=1}^{N} \frac{1}{\Gamma\left(\sigma-\beta_{j}\right)} \int_{0}^{t}(t-s)^{\sigma-\beta_{j}-1}|d(s)| d s\right) .
\end{aligned}
$$

Then the coupled system (1) has a solution.

Corollary 7. Let $f, g:[0,1] \times \mathbb{R}^{N+1} \rightarrow \mathbb{R}$ be continuous. Assume that

$\left(A^{*} 1\right)$ there exist nonnegative functions $a, c \in L^{1}[0,1]$ such that

$$
\begin{aligned}
& \left|f\left(t, x_{0}, x_{1}, \ldots, x_{N}\right)\right| \leq a(t), \\
& \left|g\left(t, x_{0}, x_{1}, \ldots, x_{N}\right)\right| \leq c(t) .
\end{aligned}
$$

Proof. In fact, let us choose $R_{0}=\max \left\{k_{1}, l_{1}\right\}$, where

$$
\begin{aligned}
& k_{1}=\max _{t \in I}( \frac{1}{\Gamma(\alpha)} \int_{0}^{t}(t-s)^{\alpha-1}|a(s)| d s \\
&\left.+\sum_{j=1}^{N} \frac{1}{\Gamma\left(\alpha-\rho_{j}\right)} \int_{0}^{t}(t-s)^{\alpha-\rho_{j}-1}|a(s)| d s\right), \\
& l_{1}=\max _{t \in I}\left(\frac{1}{\Gamma(\sigma)} \int_{0}^{t}(t-s)^{\sigma-1}|c(s)| d s\right. \\
&\left.+\sum_{j=1}^{N} \frac{1}{\Gamma\left(\sigma-\beta_{j}\right)} \int_{0}^{t}(t-s)^{\sigma-\beta_{j}-1}|c(s)| d s\right),
\end{aligned}
$$

and construct a closed ball of Banach space $X \times Y$

$$
B=\left\{(x, y) \in X \times Y:\|(x, y)\|_{X \times Y} \leq R_{0}\right\} .
$$

The rest of proof is similar to Theorem 5 .

Remark 8. The condition (A1) is weaker than (H1) and (H2). Clearly, $\phi\left(\left|x_{0}\right|,\left|x_{1}\right|, \ldots,\left|x_{N}\right|\right)$ and $\psi\left(\left|x_{0}\right|,\left|x_{1}\right|, \ldots,\left|x_{N}\right|\right)$ include $c_{0}\left|x_{0}\right|^{\gamma_{0}}+c_{1}\left|x_{1}\right|^{\gamma_{1}}+\cdots+c_{N}\left|x_{N}\right|^{\gamma_{N}}$ and $d_{0}\left|x_{0}\right|^{\theta_{0}}+$ $d_{1}\left|x_{1}\right|^{\theta_{1}}+\cdots+d_{N}\left|x_{N}\right|^{\theta_{N}}, \theta_{i}, \gamma_{i} \neq 1$ as special cases. Moreover (A1) also includes the case $\theta_{i}=1$ or/and $\gamma_{i}=1$, but (H1) and (H2) do not be allowed.

Remark 9. In Corollary 7 , for the special case $a, c \in C[0,1]$, clearly $f, g:[0,1] \times \mathbb{R}^{N+1} \rightarrow \mathbb{R}$ are continuous and bounded. This leads to the Corollary 3.1 of [12]. Therefore, Corollary 3.1 of [12] is only a special case of Corollary 7.

In the following, we focus on the uniqueness of the solution of the system (1).

Theorem 10. Let $f, g:[0,1] \times \mathbb{R}^{N+1} \rightarrow \mathbb{R}$ be continuous. Assume that

(B1) there exist nonnegative functions $a, c \in L^{1}[0,1]$ and nonnegative nondecreasing functions $\phi, \psi$ with respect to each variable $x_{i}, i=0,1,2, \ldots, N$, such that

$$
\begin{aligned}
& \left|f\left(t, u_{0}, u_{1}, \ldots, u_{N}\right)-f\left(t, v_{0}, v_{1}, \ldots, v_{N}\right)\right| \\
& \quad \leq a(t) \phi\left(\left|u_{0}-v_{0}\right|,\left|u_{1}-v_{1}\right|, \ldots,\left|u_{N}-v_{N}\right|\right), \\
& \left|g\left(t, u_{0}, u_{1}, \ldots, u_{N}\right)-g\left(t, v_{0}, v_{1}, \ldots, v_{N}\right)\right| \\
& \quad \leq b(t) \psi\left(\left|u_{0}-v_{0}\right|,\left|u_{1}-v_{1}\right|, \ldots,\left|u_{N}-v_{N}\right|\right) ;
\end{aligned}
$$

(B2) for any $s>0$,

$$
\phi(s, s, \ldots, s) \leq s, \quad \psi(s, s, \ldots, s) \leq s,
$$


Abstract and Applied Analysis

5

$$
\begin{aligned}
& k_{1}=\max _{t \in I}( \frac{1}{\Gamma(\alpha)} \int_{0}^{t}(t-s)^{\alpha-1} a(s) d s \\
&\left.+\sum_{j=1}^{N} \frac{1}{\Gamma\left(\alpha-\rho_{j}\right)} \int_{0}^{t}(t-s)^{\alpha-\rho_{j}-1} a(s) d s\right), \\
& l_{1}=\max _{t \in I}\left(\frac{1}{\Gamma(\sigma)} \int_{0}^{t}(t-s)^{\sigma-1} c(s) d s\right. \\
&\left.+\sum_{j=1}^{N} \frac{1}{\Gamma\left(\sigma-\beta_{j}\right)} \int_{0}^{t}(t-s)^{\sigma-\beta_{j}-1} c(s) d s\right) .
\end{aligned}
$$

Then coupled system (1) has a unique solution.

Proof. We prove that the operator T:X $X Y \rightarrow X \times Y$ is contraction. To do this, let $\left(x_{1}, y_{1}\right),\left(x_{2}, y_{2}\right) \in X \times Y$; we have

$$
\begin{array}{rlrl}
\left|T_{1} x_{2}(t)-T_{1} x_{1}(t)\right| & & \left.\left\|\mathscr{D}^{\beta_{N}} y_{2}-\mathscr{D}^{\beta_{N}} y_{1}\right\|\right) d s \\
=\mid \frac{1}{\Gamma(\alpha)} \int_{0}^{t}(t-s)^{\alpha-1} & \leq \frac{1}{\Gamma\left(\alpha-\rho_{j}\right)} \\
\quad \times f\left(s, y_{2}(s), \mathscr{D}^{\beta_{1}} y_{2}(s), \ldots, \mathscr{D}^{\beta_{N}} y_{2}(s)\right) d s & \times \int_{0}^{t}(t-s)^{\alpha-\rho_{j}-1} a(s) \\
-\frac{1}{\Gamma(\alpha)} \int_{0}^{t}(t-s)^{\alpha-1} & \times \frac{1}{\Gamma\left(\alpha-\rho_{j}\right)} \int_{0}^{t}(t-s)^{\alpha-\rho_{j}-1} a(s) d s\left\|y_{2}-y_{1}\right\|_{Y} .
\end{array}
$$$$
\leq \frac{1}{\Gamma(\alpha)} \int_{0}^{t}(t-s)^{\alpha-1} a(s)
$$$$
\times \phi\left(\left|y_{2}(s)-y_{1}(s)\right|,\left|\mathscr{D}^{\beta_{1}} y_{2}(s)-\mathscr{D}^{\beta_{1}} y_{1}(s)\right|, \ldots,\right.
$$$$
\left.\left|\mathscr{D}^{\beta_{N}} y_{2}(s)-\mathscr{D}^{\beta_{N}} y_{1}(s)\right|\right) d s
$$$$
\leq \frac{1}{\Gamma(\alpha)} \int_{0}^{t}(t-s)^{\alpha-1} a(s)
$$$$
\times \phi\left(\left\|y_{2}-y_{1}\right\|,\left\|\mathscr{D}^{\beta_{1}} y_{2}-\mathscr{D}^{\beta_{1}} y_{1}\right\|, \ldots,\right.
$$$$
\left.\left\|\mathscr{D}^{\beta_{N}} y_{2}-\mathscr{D}^{\beta_{N}} y_{1}\right\|\right) d s
$$$$
\leq \frac{1}{\Gamma(\alpha)} \int_{0}^{t}(t-s)^{\alpha-1} a(s)
$$$$
\times \phi\left(\left\|y_{2}-y_{1}\right\|_{Y},\left\|y_{2}-y_{1}\right\|_{Y}, \ldots,\left\|y_{2}-y_{1}\right\|_{Y}\right) d s
$$$$
\leq \frac{1}{\Gamma(\alpha)} \int_{0}^{t}(t-s)^{\alpha-1} a(s) d s\left\|y_{2}-y_{1}\right\|_{Y},
$$

$$
\begin{aligned}
& \left|\mathscr{D}^{\rho_{j}} T_{1} x_{2}(t)-\mathscr{D}^{\rho_{j}} T_{1} x_{1}(t)\right| \\
& =\mid I^{\alpha-\rho_{j}}\left(f\left(t, y_{2}(t), \mathscr{D}^{\beta_{1}} y_{2}(t), \ldots, \mathscr{D}^{\beta_{N}} y_{2}(t)\right)\right. \\
& \left.\quad-f\left(t, y_{1}(t), \mathscr{D}^{\beta_{1}} y_{1}(t), \ldots, \mathscr{D}^{\beta_{N}} y_{1}(t)\right)\right) \mid \\
& \leq \frac{1}{\Gamma\left(\alpha-\rho_{j}\right)} \\
& \quad \times \int_{0}^{t}(t-s)^{\alpha-\rho_{j}-1} \\
& \quad \times \mid f\left(t, y_{2}(t), \mathscr{D}^{\beta_{1}} y_{2}(t), \ldots, \mathscr{D}^{\beta_{N}} y_{2}(t)\right) \\
& \quad-f\left(t, y_{1}(t), \mathscr{D}^{\beta_{1}} y_{1}(t), \ldots, \mathscr{D}^{\beta_{N}} y_{1}(t)\right) \mid d s \\
& \leq \frac{1}{\Gamma\left(\alpha-\rho_{j}\right)} \\
& \quad \times \int_{0}^{t}(t-s)^{\alpha-\rho_{j}-1} a(s) \\
& \quad \times \phi\left(\left\|y_{2}-y_{1}\right\|,\left\|\mathscr{D}^{\beta_{1}} y_{2}-\mathscr{D}^{\beta_{1}} y_{1}\right\|, \ldots,\right.
\end{aligned}
$$

Thus it follows from (30) and (B2) that

$$
\begin{aligned}
& \left\|T_{1} x_{2}-T_{1} x_{1}\right\|_{X} \\
& =\max _{t \in I}\left|T_{1} x_{2}(t)-T_{1} x_{1}(t)\right| \\
& \quad+\sum_{j=1}^{N} \max _{t \in I}\left|\mathscr{D}^{\rho_{j}}\left(T_{1} x_{2}(t)-T_{1} x_{1}(t)\right)\right| \\
& \leq\left(\frac{1}{\Gamma(\alpha)} \int_{0}^{t}(t-s)^{\alpha-1} a(s) d s\right. \\
& \left.\quad+\sum_{j=1}^{N} \frac{1}{\Gamma\left(\alpha-\rho_{j}\right)} \int_{0}^{t}(t-s)^{\alpha-\rho_{j}-1} a(s) d s\right)\left\|y_{2}-y_{1}\right\|_{Y} \\
& \leq k_{1}\left\|y_{2}-y_{1}\right\|_{Y} \cdot
\end{aligned}
$$


Similarly, we can get

$$
\begin{aligned}
& \left|T_{1} y_{2}(t)-T_{1} y_{1}(t)\right| \\
& \leq \frac{1}{\Gamma(\sigma)} \int_{0}^{t}(t-s)^{\sigma-1} c(s) d s\left\|x_{2}-x_{1}\right\|_{X}, \\
& \left|\mathscr{D}^{\beta_{j}} T_{1} y_{2}(t)-\mathscr{D}^{\beta_{j}} T_{1} y_{1}(t)\right| \\
& \leq \frac{1}{\Gamma\left(\sigma-\beta_{j}\right)} \int_{0}^{t}(t-s)^{\sigma-\beta_{j}-1} c(s) d s\left\|x_{2}-x_{1}\right\|_{X}, \\
& \left\|T_{1} y_{2}-T_{1} y_{1}\right\|_{Y} \\
& =\max _{t \in I}\left|T_{1} y_{2}(t)-T_{1} y_{1}(t)\right| \\
& \quad+\sum_{j=1}^{N} \max _{t \in I}\left|\mathscr{D}^{\rho_{j}}\left(T_{1} y_{2}(t)-T_{1} y_{1}(t)\right)\right| \\
& \leq\left(\frac{1}{\Gamma(\sigma)} \int_{0}^{t}(t-s)^{\sigma-1} c(s) d s\right. \\
& \leq l_{1}\left\|x_{2}-x_{1}\right\|_{X} \cdot \\
& \left.+\sum_{j=1}^{N} \frac{1}{\Gamma\left(\sigma-\beta_{j}\right)} \int_{0}^{t}(t-s)^{\sigma-\beta_{j}-1} c(s) d s\right)\left\|x_{2}-x_{1}\right\|_{X}
\end{aligned}
$$

Hence, for the Euclidean distance $d$ on $\mathbb{R}^{2}$, we get

$$
\begin{aligned}
d(T & \left.\left(x_{2}, y_{2}\right), T\left(x_{1}, y_{1}\right)\right) \\
& =\sqrt{\left\|T_{1} x_{2}-T_{1} x_{1}\right\|_{X}^{2}+\left\|T_{1} y_{2}-T_{1} y_{1}\right\|_{Y}^{2}} \\
& \leq \sqrt{k_{1}^{2}\left\|x_{2}-x_{1}\right\|_{X}^{2}+l_{1}^{2}\left\|y_{2}-T_{1}\right\|_{Y}^{2}} \\
& \leq \sqrt{\max \left\{k_{1}^{2}, l_{1}^{2}\right\}} \sqrt{\left\|x_{2}-x_{1}\right\|_{X}^{2}+\left\|y_{2}-T_{1}\right\|_{Y}^{2}} \\
& =\sqrt{\max \left\{k_{1}^{2}, l_{1}^{2}\right\}} d\left(\left(x_{2}, y_{2}\right),\left(x_{1}, y_{1}\right)\right) .
\end{aligned}
$$

Thus $T$ is a contraction since $\sqrt{\max \left\{k_{1}^{2}, l_{1}^{2}\right\}}<1$.

By Banach contraction principle, $T$ has a unique fixed point, which is a solution of the coupled system (1). The proof is completed.

An Example. Consider the existence of solutions for the following coupled system of multiterm nonlinear fractional differential equations:

$$
\begin{aligned}
&-\mathscr{D}^{3.5} x(t)= \frac{t}{6.08}+\frac{1}{25.26}\left[y(t)+e^{\left(\mathscr{D}^{0.8} y(t)\right)}\right], \\
&-\mathscr{D}^{3.5} x(0)=0, \quad i=1,2, \ldots, 4, \\
& \mathscr{D}^{4.2} y(t)= \frac{10000}{5501}\left[t^{-1 / 2} x^{0.2}(t)+t^{2}\left(\mathscr{D}^{0.5} x(t)\right)^{0.5}\right], \\
& \mathscr{D}^{4.2-j} y(0)=0, \quad j=1,2, \ldots, 5,
\end{aligned}
$$

where $t \in(0,1]$.
Let

$$
\begin{gathered}
f\left(t, x_{0}, x_{1}\right)=\frac{t}{6.08}+\frac{1}{25.26}\left[x_{0}+e^{x_{1}}\right], \\
g\left(t, x_{0}, x_{1}\right)=t^{-1 / 2} x_{0}^{0.2}+t^{2} x_{1}^{0.5},
\end{gathered}
$$

and choose

$$
\begin{gathered}
a(t)=\frac{t}{6.08}, \quad b(t)=\frac{1}{25.26}, \\
\phi\left(x_{0}, x_{1}\right)=x_{0}+e^{x_{1}}, \quad c(t)=0, \\
d(t)=\frac{10000}{5501}\left[t^{-1 / 2}+t^{2}\right] \\
\psi\left(x_{0}, x_{1}\right)=x_{0}^{0.2}+x_{1}^{0.5} .
\end{gathered}
$$

Then

$$
\begin{aligned}
& f\left(t, x_{0}, x_{1}\right) \leq a(t)+b(t) \phi\left(x_{0}, x_{1}\right), \\
& g\left(t, x_{0}, x_{1}\right) \leq c(t)+d(t) \psi\left(x_{0}, x_{1}\right) ;
\end{aligned}
$$

consequently, (A1) holds.

In the following, we check the condition (A1). Since

$$
\begin{aligned}
k_{1}= & \max \left(\frac{1}{\Gamma(3.5)} \int_{0}^{t} \frac{(t-s)^{2.5} s}{6.08} d s+\frac{1}{\Gamma(3)} \int_{0}^{t} \frac{(t-s)^{2} s}{6.08} d s\right) \\
= & 0.01, \\
k_{2}= & \max \left(\frac{1}{\Gamma(3.5)} \int_{0}^{t} \frac{(t-s)^{2.5}}{25.26} d s+\frac{1}{\Gamma(3)} \int_{0}^{t} \frac{(t-s)^{2}}{25.26} d s\right) \\
= & 0.01, \\
l_{1}= & 0, \\
l_{2}= & \frac{10000}{5501} \\
& \times \max \left(\frac{1}{\Gamma(4.2)} \int_{0}^{t}(t-s)^{3.2}\left(s^{-1 / 2}+s^{2}\right) d s\right. \\
& \left.\quad+\frac{1}{\Gamma(3.4)} \int_{0}^{t}(t-s)^{2.4}\left(s^{-1 / 2}+s^{2}\right) d s\right)=1,
\end{aligned}
$$

take $R_{0}=5$; we have

$$
\begin{aligned}
& \phi\left(R_{0}, R_{0}\right)=R_{0}+e^{R_{0}}=5+e^{5} \\
&=153.44<\frac{R_{0}-k_{1}}{k_{2}}=\frac{5-0.01}{0.01}=499, \\
& \psi\left(R_{0}, R_{0}\right)=R_{0}^{0.2}+R_{0}^{0.5}=5^{0.2}+5^{0.5} \\
&=3.6158<\frac{R_{0}-l_{1}}{l_{2}}=5
\end{aligned}
$$

which implies that (A2) is satisfied. Hence, by Theorem 5, the coupled system of fractional differential equation (34) has a solution. 
Remark 11. In the coupled system of fractional differential equation (34), the nonlinear function $f$ involves exponential growth, but the results of [12] are only allowed to be power growth; that is, (34) cannot be solved by using the results of [12]. So the results obtained in this paper give a significant improvement of the previous work in [12].

\section{Acknowledgments}

The authors would like to express their sincere gratitude to the anonymous reviewers and academic editor for a number of valuable comments and suggestions. The authors were supported financially by the Natural Science Foundation of Shandong Province of China (ZR2010AM022).

\section{References}

[1] I. Podlubny, Fractional Differential Equations, Mathematics in Science and Engineering, Academic Press, New York, NY, USA, 1999.

[2] K. Miller and B. Ross, An Introduction to the Fractional Calculus and Fractional Differential Equations, John Wiley \& Sons, New York, NY, USA, 1993.

[3] S. Samko, A. Kilbas, and O. Marichev, Fractional Integrals and Derivatives, Gordon and Breach Science, Yverdon, Switzerland, 1993.

[4] M. Jia, X. Liu, and X. Gu, "Uniqueness and asymptotic behavior of positive solutions for a fractional-order integral boundary value problem," Abstract and Applied Analysis, vol. 2012, Article ID 294694, 21 pages, 2012.

[5] X. Zhang, L. Liu, and Y. Wu, "The uniqueness of positive solution for a singular fractional differential system involving derivatives," Communications in Nonlinear Science and Numerical Simulation, vol. 18, no. 6, pp. 1400-1409, 2013.

[6] B. Ahmad and J. Nieto, "Existence results for a coupled system of nonlinear fractional differential equations with three-point boundary conditions," Computers \& Mathematics with Applications, vol. 58, no. 9, pp. 1838-1843, 2009.

[7] X. Zhang, L. Liu, B. Wiwatanapataphee, and Y. Wu, "Positive solutions of eigenvalue problems for a class of fractional differential equations with derivatives," Abstract and Applied Analysis, vol. 2012, Article ID 512127, 16 pages, 2012.

[8] V. Daftardar-Gejji, "Positive solutions of a system of nonautonomous fractional differential equations," Journal of Mathematical Analysis and Applications, vol. 302, no. 1, pp. 56-64, 2005.

[9] X. Zhang and Y. Han, "Existence and uniqueness of positive solutions for higher order nonlocal fractional differential equations," Applied Mathematics Letters, vol. 25, no. 3, pp. 555-560, 2012.

[10] H. Salem, "On the existence of continuous solutions for a singular system of non-linear fractional differential equations," Applied Mathematics and Computation, vol. 198, no. 1, pp. 445$452,2008$.

[11] X. Su, "Boundary value problem for a coupled system of nonlinear fractional differential equations," Applied Mathematics Letters, vol. 22, no. 1, pp. 64-69, 2009.

[12] S. Sun, Q. Li, and Y. Li, "Existence and uniqueness of solutions for a coupled system of multi-term nonlinear fractional differential equations," Computers \& Mathematics with Applications, vol. 64, no. 10, pp. 3310-3320, 2012.
[13] X. Zhang, L. Liu, Y. Wu, and Y. Lu, "The iterative solutions of nonlinear fractional differential equations," Applied Mathematics and Computation, vol. 219, no. 9, pp. 4680-4691, 2013.

[14] X. Zhang, L. Liu, and Y. Wu, "The eigenvalue problem for a singular higher order fractional differential equation involving fractional derivatives," Applied Mathematics and Computation, vol. 218, no. 17, pp. 8526-8536, 2012.

[15] C. S. Goodrich, "Existence of a positive solution to systems of differential equations of fractional order," Computers \& Mathematics with Applications, vol. 62, no. 3, pp. 1251-1268, 2011.

[16] X. Zhang, L. Liu, and Y. Wu, "Multiple positive solutions of a singular fractional differential equation with negatively perturbed term," Mathematical and Computer Modelling, vol. 55, no. 3-4, pp. 1263-1274, 2012. 


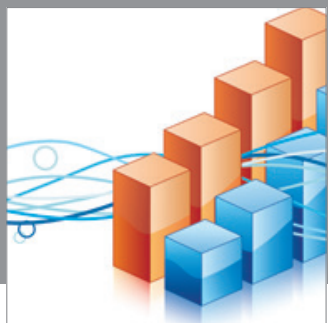

Advances in

Operations Research

mansans

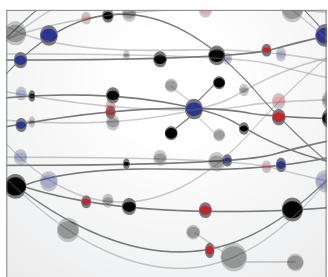

The Scientific World Journal
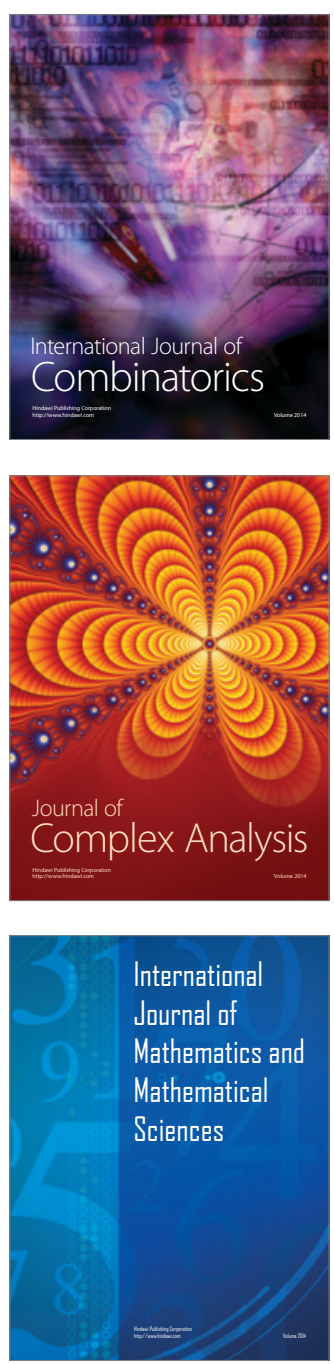
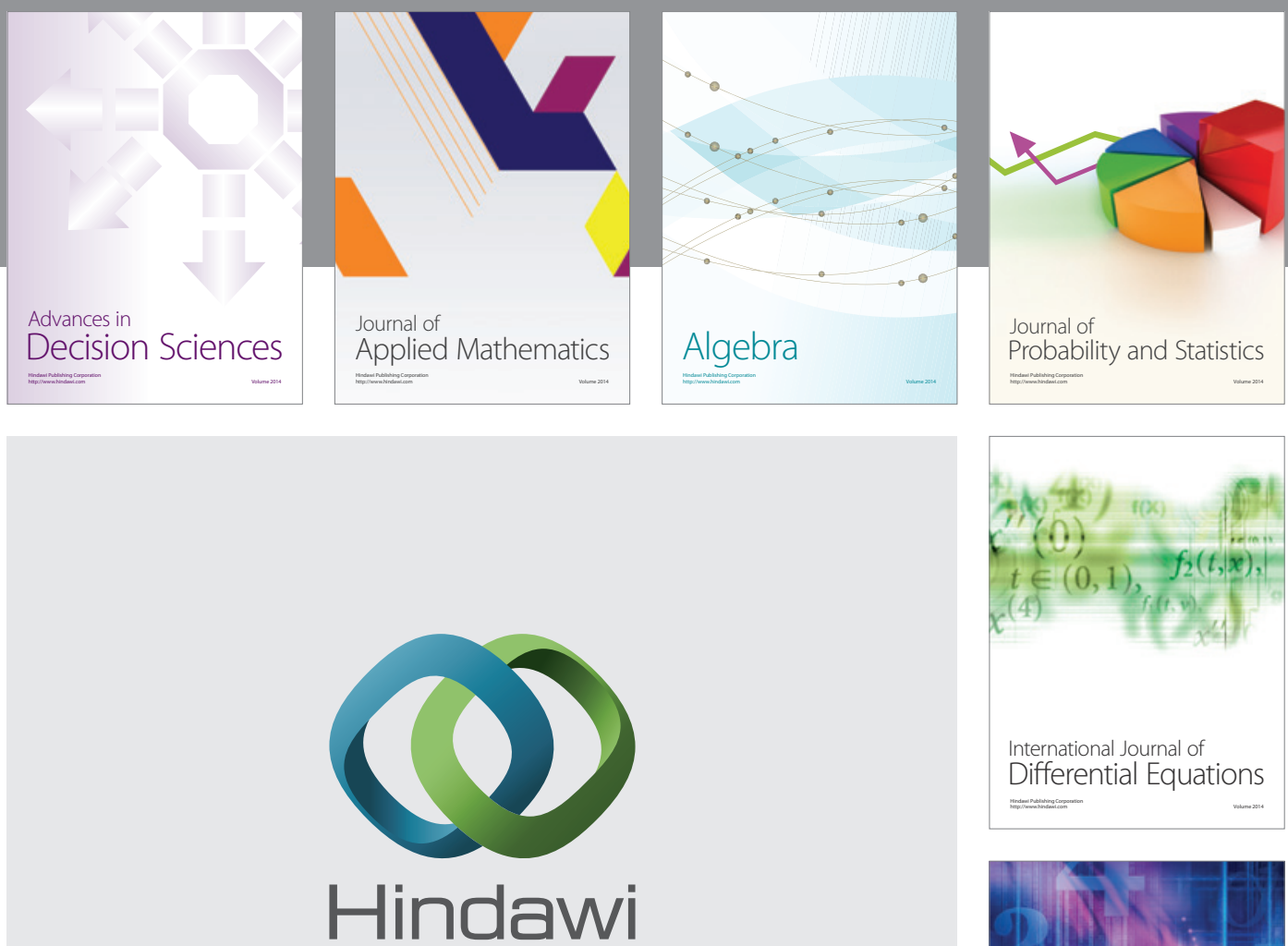

Submit your manuscripts at http://www.hindawi.com
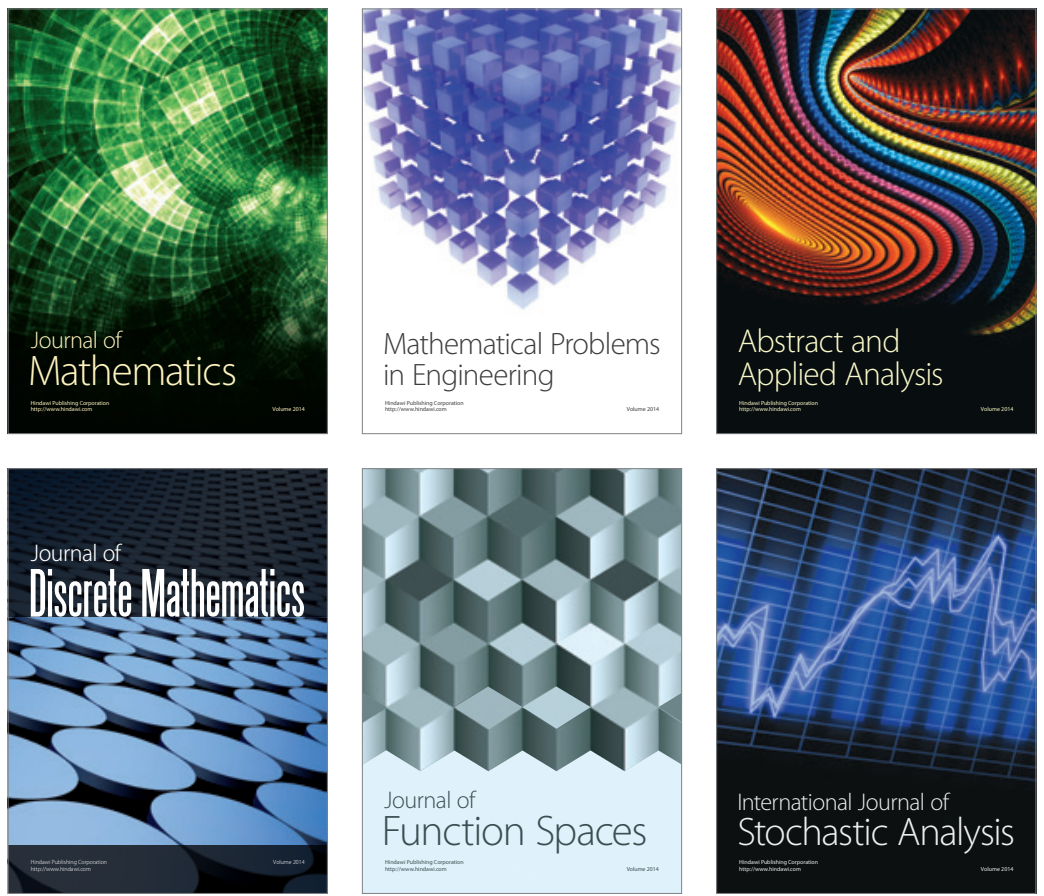

Journal of

Function Spaces

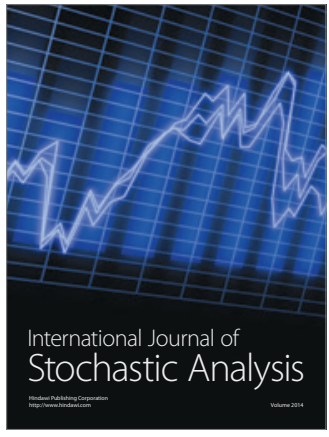

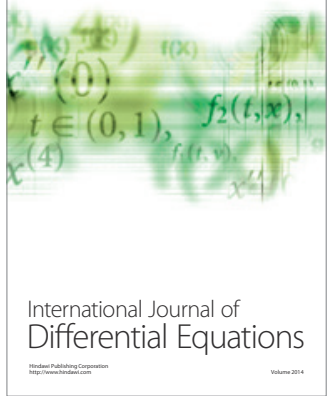
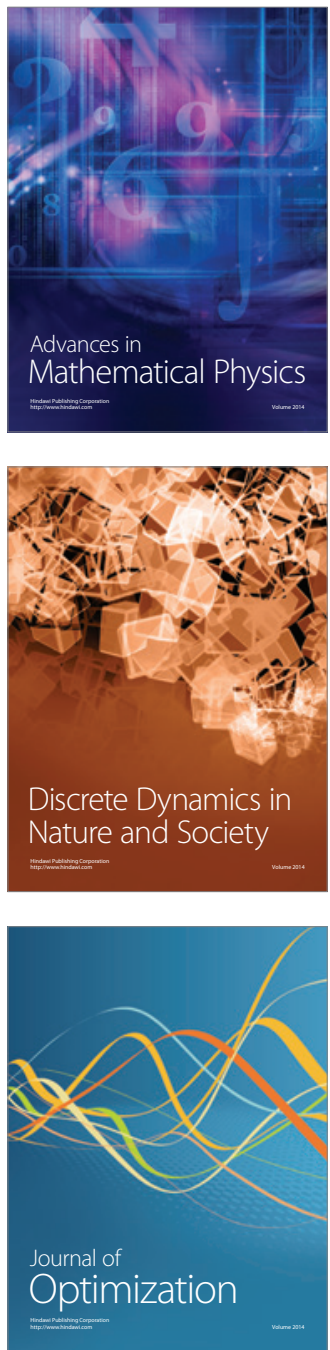